\title{
The Stability of Gold Nanoparticles in Liquid Scanning Transmission Electron Microscopy Experiments Studied under Varied Conditions.
}

\author{
Andreas Verch ${ }^{1}$, Justus Hermannsdörfer ${ }^{1}$ and Niels de Jonge ${ }^{1,2}$. \\ ${ }^{1 .}$ INM - Leibniz Institute for New Materials, Campus D2 2, 66123 Saarbrücken, Germany \\ 2. Department of Physics, University of Saarland, Campus A5 1, 66123 Saarbrücken, Germany
}

Studying dynamical processes such as the self-assembly of nano-particles in liquid with nanometer resolution is possible using a recently established technology for transmission electron microscopy (TEM) in liquid [1]. The sample containing liquid and solid parts are placed in a microfluidic chamber between two electron transparent membranes. The principal setup is schematically shown in Fig. 1. However, interactions between the liquids and the electron beam dramatically increase the complexity of the system and complicate the analysis of the observed processes. High energy electrons excite electronic states in the specimen and the ample liquid resulting in the generation of highly reactive, transient species such as solvated electrons, hydrogen-, and hydroxide radicals. Their appearance initiates a cascade of reactions in which various other powerful oxidizing and reducing agents are formed. These species are often able to attack the solid specimen chemically, which might change its surface properties or actually dissolve it. In many cases this behavior is not desired and it might even render the experiment useless. In order to design experiments resembling the unperturbed specimen as closely as possible, it is thus essential to improve our understanding of the processes occurring in liquid electron microscopy experiments, and to learn how to reduce the impact of the electron beam on the specimen.

In a liquid TEM experiment, the steady state concentrations of the different reactive species and even the $\mathrm{pH}$ are significantly influenced by parameters such as the initial $\mathrm{pH}$ of the solution and the imaging conditions, e.g. the electron dose, as simulations based on equilibrium and rate constants of the aforementioned transient and other involved species have suggested.[2]. Hence, a $\mathrm{pH}$-sensitive experiment, for example, might have an unexpected outcome, since the $\mathrm{pH}$ of the solution may be changed as a result of the electron beam interactions. In this work, we studied the stability of gold nanoparticles in a liquid scanning TEM experiment as function of several varied solution parameters, and imaging conditions. We varied the $\mathrm{pH}$, the sodium chloride concentration, the ionic strength of the aqueous phase, and the electron dose introduced into the solution. Our experiments were conducted using a liquid TEM holder (Poseidon, Protochips, NC, USA) in a $\mathrm{C}_{\mathrm{S}}$-corrected STEM/TEM (ARM200f, JEOL. Japan) at $200 \mathrm{kV}$ acceleration voltage. The microscope was operated in scanning mode in order to obtain a better handle on the doses introduced into the area observed.

Our experiments showed that the addition of sodium chloride strongly influenced the stability of the gold nanoparticles. At neutral $\mathrm{pH}$ conditions the addition of chloride ions decreased the redox potential for gold oxidation, leading to an accelerated dissolution of the nanoparticles. The influence of the $\mathrm{pH}$ on the underlying reactions became obvious when the $\mathrm{pH}$ is changed. While a decrease of the $\mathrm{pH}$ merely the gold dissolution rate rates, high $\mathrm{pH}$ values significantly altered the behavior of the gold nanoparticles in presence of chloride ions. Instead of dissolving, the nanoparticles drew nearer and eventually coalesced within a few second. This observations confirmed the complex interplay of oxidizing and reducing species with the original nanoparticles, the dissolved-, and the precipitated gold. Video stills of experiments at different $\mathrm{pH}$ conditions illustrating this behavior are shown in Fig. 2.

References: 
[1] N. de Jonge and F. M. Ross, Nature Nanotechnology 6 (2011), pp. 695.

[2] N. M. Schneider et al., The Journal of Physical Chemistry C 118 (2014), pp. 22373.

We thank Protochips Inc, NC, USA for providing the microchips and the Liquid STEM specimen holder. We thank E. Arzt for his support through INM. Research in part supported by the Leibniz Competition 2014.

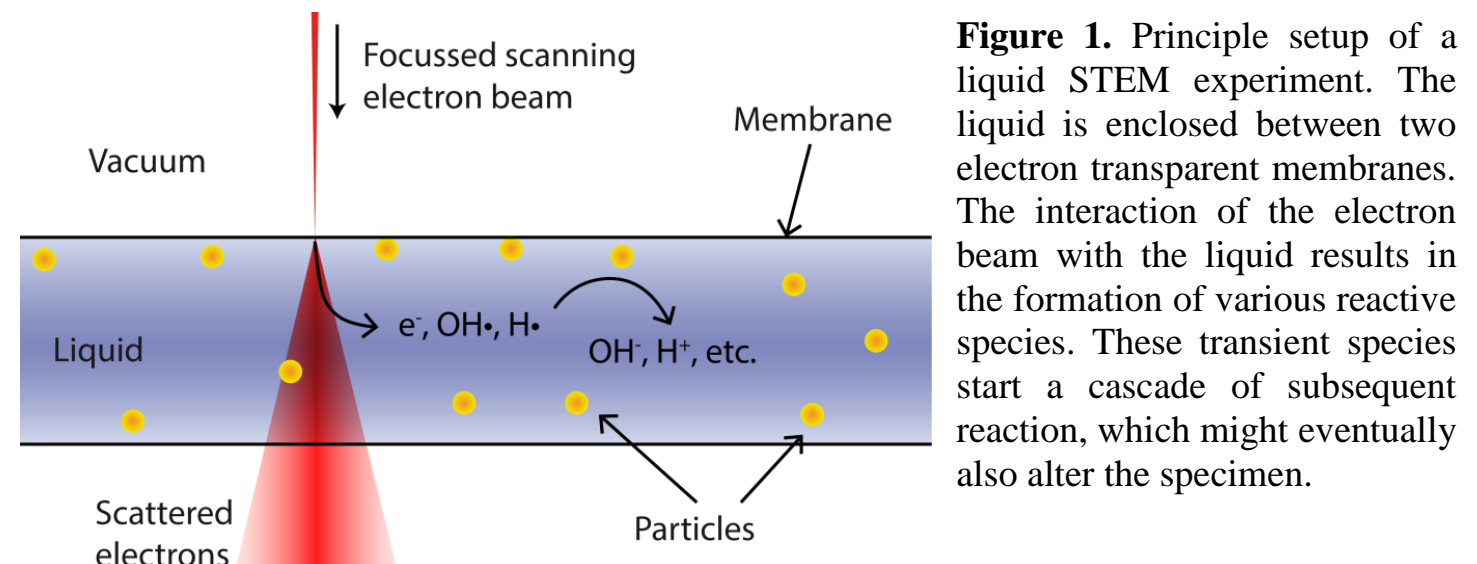

Figure 1. Principle setup of a liquid STEM experiment. The liquid is enclosed between two The inte beam with the liquid results in the formation of various reactive species. These transient species start a cascade of subsequent might eventually electrons field (HAADF) detector
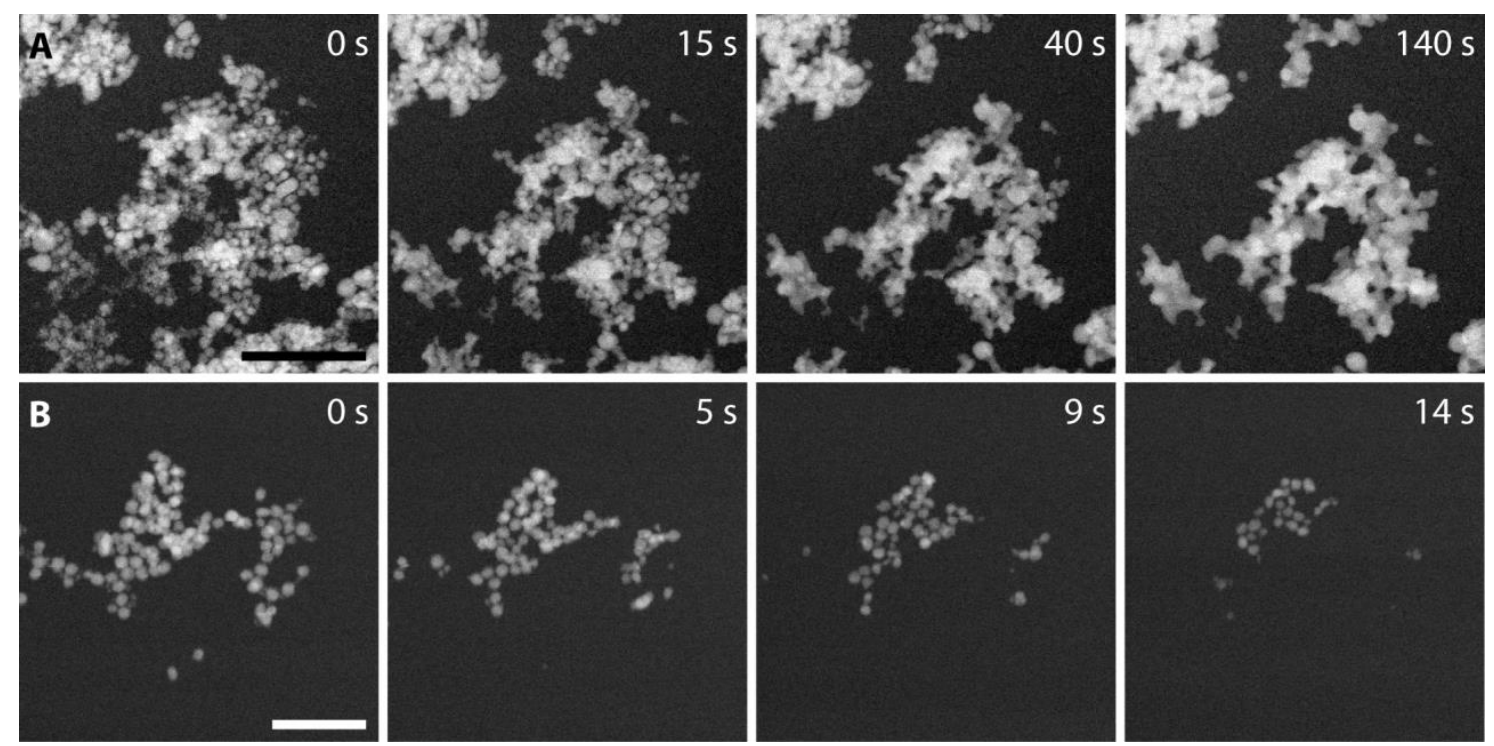

Figure 2. Stills of videos recorded with Liquid STEM showing the behavior of gold nanoparticles at different solution $\mathrm{pH}$ in the presence of $2 \mathrm{~mol} \mathrm{~L}^{-1} \mathrm{NaCl}$. (A) At $\mathrm{pH} 12$ the gold nanoparticles approach each other and eventually grow together. The electron dose rate per image was $12 \mathrm{e}^{-} \AA^{-1} \mathrm{~s}^{-1}$. (B) The gold nanoparticles rapidly dissolve at $\mathrm{pH}$ 7. Electron dose: $3 \mathrm{e}^{-} \AA^{-1} \mathrm{~s}^{-1}$. Both scale bars represent $200 \mathrm{~nm}$. 